\title{
Computed Tomography-Guided Virtual Stereotactic Puncture and Catheter Drainage for a Brainstem Pontine Hemorrhage: A Case Report
}

\author{
Gang Yang*, Shaojun Yang, Junjie Lv, Chenbing Wang \\ Department of Neurosurgery, Zhuji Affiliated Hospital of Shaoxing University, Shaoxing, China \\ Email:^tekeyang@sina.com
}

How to cite this paper: Yang, G., Yang, S.J., Lv, J.J. and Wang, C.B. (2020) Computed Tomography-Guided Virtual Stereotactic Puncture and Catheter Drainage for a Brainstem Pontine Hemorrhage: A Case Report. Case Reports in Clinical Medicine, 9, 223-227.

https://doi.org/10.4236/crcm.2020.98031

Received: June 22, 2020

Accepted: August 3, 2020

Published: August 6, 2020

Copyright $\odot 2020$ by author(s) and Scientific Research Publishing Inc. This work is licensed under the Creative Commons Attribution International License (CC BY 4.0).

http://creativecommons.org/licenses/by/4.0/

(c) (i) Open Access

\begin{abstract}
Background: Patients with severe hypertensive brainstem hemorrhages have poor prognoses if they only receive conservative medical management. In contrast, aggressive operative interventions may decrease the morbidity and mortality in such patients. These operative treatments include craniotomy for evacuation of the hematoma, stereotactic positioning, and neuronavigational-guided hemorrhage puncture and drainage. Here, we report a novel and relatively simple procedure to achieve satisfactory outcomes in a patient with a brainstem pontine hemorrhage. Case Presentation: A 53-year-old man who was diagnosed with brainstem pontine hemorrhage. On hospital day 6, he underwent CT-guided, virtual stereotactic puncture and catheter drainage of this brainstem pontine hemorrhage. Medical treatments were continued after this procedure. On postoperative day 16 (hospital day 22), the patient was discharged from the hospital, awake and able to answer questions appropriately. Muscle strengths were grades V and IV for the left and right extremities, respectively. The patient was continued with active rehabilitation and achieved a Barthel index of 85 points at one month after the percutaneous drainage procedure. Conclusion: CT-guided, virtual stereotactic percutaneous transcranial puncture and catheter drainage for brainstem pontine hemorrhages has obvious potential advantages and offers a possible alternative to achieve the best outcomes with minimal operative trauma compared to open microcraniotomy.
\end{abstract}

\section{Keywords}

Computed Tomography-Guided, Stereotactic, Catheter Drainage, Pontine Hemorrhage 


\section{Background}

Patients with severe hypertensive brainstem hemorrhages have poor prognoses if they only receive conservative medical management. In contrast, aggressive operative interventions may decrease the morbidity and mortality in such patients. These operative treatments include craniotomy for evacuation of the hematoma, stereotactic positioning, and neuronavigational-guided hemorrhage puncture and drainage. Here, we report a novel and relatively simple procedure to achieve satisfactory outcomes in a patient with a brainstem pontine hemorrhage.

\section{Case Presentation}

A 55-year-old male was admitted to our hospital on September 29, 2019, due to the sudden onset of a coma of $2 \mathrm{~h}$ duration. He had a history of hypertension but was non-compliant with taking his medication for this condition. The patient had no reported prior history of neurosurgical intervention. The admission evaluation documented a comatose man with a Glasgow Coma Scale (GCS) score of E1TV3. The patient's pupils were unresponsive to light and had diameters of $1 \mathrm{~mm}$ and $2 \mathrm{~mm}$ for the right and left eyes, respectively. His vital signs were as follows: heart rate of 110 beats/min, blood pressure of 175/100 $\mathrm{mmHg}$, respiratory rate of $16 \mathrm{breaths} / \mathrm{min}$, and a temperature of $38.5^{\circ} \mathrm{C} . \mathrm{He}$ withdrew his left but not right extremity to pain stimulation. A pathologic Babinski sign was induced bilaterally. Computed tomography (CT) on the day of admission showed a brainstem pontine hemorrhage (Figure 1(a)). He was treated with mechanical ventilation, conservative medical management.

On hospital day 6, the patient was still in a coma with a persistent GCS score of E1TV3. He still required mechanical ventilation. Muscle strength tests revealed horizontal movements to the left extremity and slight flexion to the right extremity with pain stimulations. On the same day, a CT was repeated and showed a brainstem pontine hemorrhage (Figure 1(b)), for which he underwent CT-guided, virtual stereotactic puncture and catheter drainage of this brainstem pontine hemorrhage.

\section{Surgical Procedure}

The baseline of the brain CT scan was from the supraorbital ridge to $2 \mathrm{~cm}$ below the external occipital protuberance. The middle level of the pontine hemorrhage was selected as the horizontal positioning plane (Figure $1(\mathrm{c})$ ). The central location of the brainstem hemorrhage is labeled as point B in the CT images. The proximal skin puncture site was inferior and posterior to the occipital mastoid; the pathway of puncture was aimed at the center point of brainstem hemorrhage and was designated as point A on the CT images. The distal site was lateral to the supraorbital ridge in the contralateral temporal area and is designated as point $\mathrm{C}$ and could be adjusted by the site labeled as "MARK" (Figure 1(d)). Point A and point $B$ were marked on the head. The distances between the points were meas- 
ured and labeled as $\mathrm{AB}, \mathrm{DA}$, and EC. Based on stereotactic theory, horizontal and vertical lines for punctures (horizontal and vertical planes) were marked on the skin with respect to the above-mentioned puncture lines. The depth of puncture (distance of $\mathrm{AB}$ ) was then calculated. According to the horizontal and vertical planes, a drainage catheter with a diameter of $3 \mathrm{~mm}$ was inserted along the puncture path into the hemorrhage. Approximately $3 \mathrm{~mL}$ of blood was obtained using a slight negative-suction pressure. Extraventricular drainage was also applied simultaneously. A postoperative head CT showed a substantial decrease in the size of the pontine hemorrhage, with a satisfactory positioning of the drainage catheter (Figure 2(a), Figure 2(b)).

Medical treatments were continued after this procedure. The drainage catheter was removed on postoperative day 2 when there is no bloody fluid flowing out. Repeat CT images on postoperative days 6 and 14 (hospital days 12 and 20) are shown in Figure 2(c), Figure 2(d). On postoperative day 16 (hospital day 22), the patient was discharged from the hospital, awake and able to answer questions appropriately. Muscle strengths were grades V and IV for the left and right extremities, respectively. The patient was continued with active rehabilitation and achieved a Barthel index of 85 points at one month after the percutaneous drainage procedure.

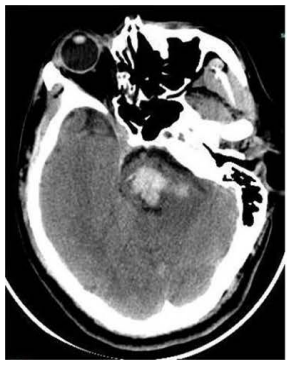

(a)

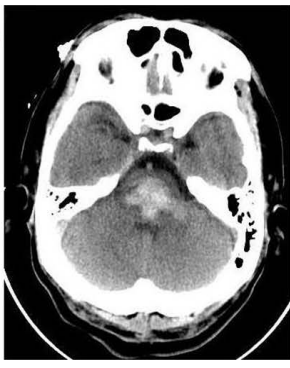

(b)

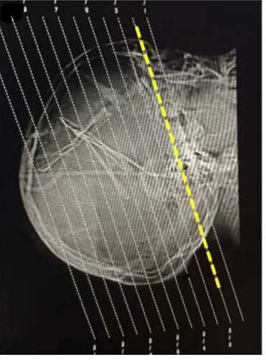

(c)

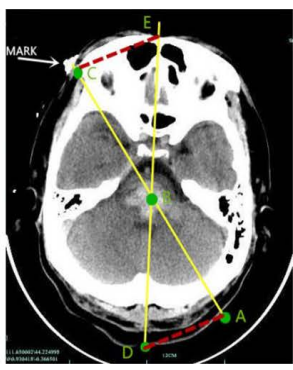

(d)

Figure 1. CT on admission (a), CT before operation (b), the baseline of the brain CT scan was from the supraorbital ridge to $2 \mathrm{~cm}$ below the external occipital protuberance (c), the pathway of puncture $A C$ was aimed at the center point of brainstem hemorrhage, The depth of puncture is $A B(d)$.

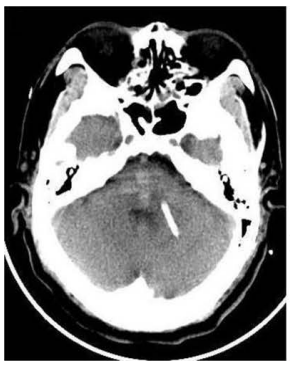

(a)

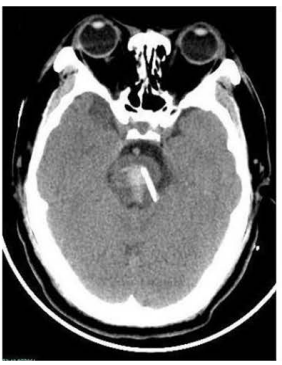

(b)

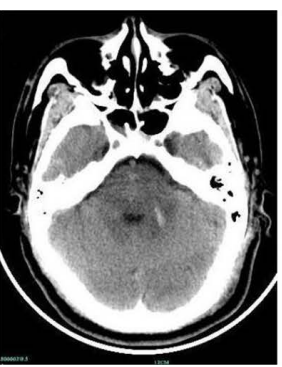

(c)

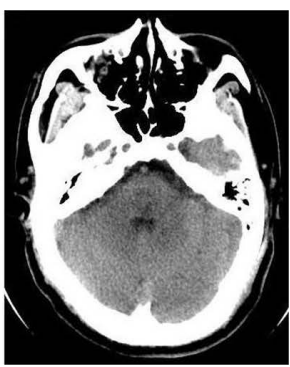

(d)

Figure 2. A postoperative head CT showed a substantial decrease in the size of the pontine hemorrhage, with a satisfactory positioning of the drainage catheter (a) (b). Repeat CT images on postoperative days 6 and 14 (hospital days 12 and 20) are shown in (c) (d). 


\section{Discussion}

Intracranial hemorrhage is a serious but common disorder. Approximately $10 \%$ of intracranial hemorrhages are brainstem hemorrhages, which have an overall mortality rate of $40 \%-50 \%$ [1]. Patients with more than $5 \mathrm{ml}$ of bleeding are considered to have a severe hemorrhage. Such patients usually have poor outcomes, with a mortality rate as high as $80 \%-100 \%$ [2]. Unfortunately, medical conservation treatments commonly result in extremely poor prognoses in these patients.

While operative treatment for severe hypertensive brainstem hemorrhages have achieved promising results [3] [4], the microsurgical craniotomy techniques to evacuate such hemorrhages are complicated and can cause significant surgical trauma. The high costs and need for expertise for stereotactic and neuronavigational interventions also limit their clinical applications. Hence, the development, adoption, and experience with these new techniques in primary hospitals are limited and therefore reaching an optimal medical benefit/expense ratio has been a considerable challenge for neurosurgeons. Nevertheless, appropriate and relatively straightforward training of neurosurgeons has led to successful mastery of the technique of lateral ventricular puncture and drainage. As such, we wondered whether a percutaneous transcranial puncture method could be developed and implemented to treat brainstem hemorrhages based on the findings of a routine head CT. From our own accumulated clinical experience, in addition to referencing the principle of stereotactic intracranial localization of lesions, we hypothesized that accomplishing this goal of percutaneous evacuation of a localized hematoma was imminently feasible. For the patient in our present case report, the operation was relatively straightforward, required only the guidance of routine head CT images, and was easily accomplished. The duration of the operation was only half an hour. The location of the hematoma was accurately identified, the hematoma evacuated, a drain left in place, and the recovery and outcome of the patient was satisfactory.

In patients with brainstem edema or hydrocephalus, lateral ventricle drainage can be performed simultaneously. This combined approach may not only decrease markedly the increased intracranial pressure, but may also induce drainage of the hematoma into the ventricle. In addition, any postoperative residual hematoma can be treated with the administration of a small amount of urokinase through an indwelling drainage catheter into the hematoma. After confirming the evacuation of the hematoma on a postoperative CT, the drainage catheter can be removed. Additionally, conventional treatments-such as mechanical ventilation, fluid control to decrease the intracranial pressure, optimal control of the blood pressure, analgesia/sedation, and nutritional support-should also be applied for comprehensive management of patients with intracranial hemorrhage treated by such a percutaneous, transcranial approach.

In summary, minimally invasive, stereotactically directed percutaneous, transcranial puncture and drainage appears to be a promising approach to treating 
localized hypertensive brainstem hemorrhages. Before using this approach, however, brainstem cavernous hemangiomas, hemorrhage secondary to a tumor, and aneurysmal hemorrhages should be excluded preoperatively. The proximal skin puncture site that is inferior and posterior to the occipital mastoid should avoid the transverse sinus to maximize the safety of the approach.

\title{
4. Conclusion
}

CT-guided, virtual stereotactic percutaneous transcranial puncture and catheter drainage for brainstem pontine hemorrhages has obvious potential advantages and offers a possible alternative to achieve the best outcomes with minimal operative trauma compared to open microcraniotomy. In addition, this procedure does not require any special equipment, which can facilitate its wide adoption in many primary hospitals. This procedure is suitable for patients with severe localized hypertensive brainstem hemorrhages and offers novel potential advance for managing patients with hypertensive brainstem hemorrhages.

\section{Conflicts of Interest}

The authors declare no conflicts of interest regarding the publication of this paper.

\section{References}

[1] Wessels, T., Moller-Hatmann, W., Noth, J., et al. (2004) CT Findings and Clinical Features as Markers for Patient Outcome in Primary Pontine Hemorrhage. American Journal of Neuroradiolog, 25, 257-260.

[2] Indredavik, B., Bakke, F., Slordahl, S.A., et al. (1999) Stroke Unit Treatment. 10-Year Follow-Up. Stroke, 30, 1524-1527. https://doi.org/10.1161/01.STR.30.8.1524

[3] Ichimura, S., Bertalanffy, H., Nakaya, M., et al. (2018) Surgical Treatment for Primary Brainstem Hemorrhage to Improve Postoperative Functional Outcomes. World Neurosurgery, 120, e1289-e1294. https://doi.org/10.1016/j.wneu.2018.09.055

[4] Wang, Q., Guo, W., Liu, Y., et al. (2019) Application of a3D-Printed Navigation Mold in Puncture Drainage for Brainstem Hemorrhage. Journal of Surgical Research, 245, 99-106. https://doi.org/10.1016/j.jss.2019.07.026

\author{
Abbreviations \\ GCS: Glasgow Coma Scale \\ CT: Computed Tomography
}

